\title{
Methanol reactivity on silica-supported ceria nanoparticles
}

\author{
John J. Uhlrich, Bing Yang, and Shamil Shaikhutdinov*
}

\begin{abstract}
Ceria $\left(\mathrm{CeO}_{2}\right)$ has been used in a number of catalytic processes, either as a support or promoter. For a better understanding of the factors that control the reactivity of ceria, we have used well-ordered $\mathrm{CeO}_{2}(111)$ films and ceria nanoparticles supported on an ordered $\mathrm{SiO}_{2}$ film, as model catalysts. The systems were examined in the dehydrogenation of methanol to formaldehyde as a test reaction by using the techniques of infrared spectroscopy and temperature programmed desorption. The results revealed low-temperature reactivity (below $450 \mathrm{~K}$ ) for supported ceria particles that is not present on ordered films, which show reactivity at $565 \mathrm{~K}$. The results indicate that low-coordinated sites play an important role in the methanol reactivity on ceria.
\end{abstract}

Keywords: Model catalysts; Ceria; Methanol.

* Corresponding author. E-mail: shaikhutdinov@fhi-berlin.mpg.de 


\section{Introduction}

Ceria-containing catalysts are used for a number of industrially important catalytic processes [1-3]. A key feature of ceria in the catalysts is its capacity to readily donate oxygen to the catalytic species, which renders ceria as a good catalyst and/or promoter in many oxidation reactions. The oxidative dehydrogenation $(\mathrm{ODH})$ of methanol to formaldehyde is often used as a test reaction for selective oxidation catalysts [4]. The commonly accepted mechanism for $\mathrm{ODH}$ on oxides begins with the adsorption of methanol on a cation-anion (or Lewis acid-base) pair. The methoxy species then forms on the cation, and the methanol proton adsorbs on a lattice oxygen to form a hydroxyl group. Then, a hydrogen atom is abstracted from the methoxy group to a second lattice oxygen and formaldehyde is produced, which desorbs from the surface. The hydroxyl groups recombine to form water that desorbs and thus leaves an oxygen vacancy behind, which can subsequently be replenished from molecular oxygen in the ambient. Following this mechanism it is expected that the reactivity of the oxide surface will critically depend on the oxygen-vacancy-formation energy, which is therefore considered as a suitable descriptor for ODH reactions $[5,6]$. This may, in turn, depend on the crystal plane of the oxide. Obviously, the situation becomes more complicated if the oxide is highly dispersed to form nanoparticles, which expose different surfaces as well as substantial amounts of lowcoordinated sites.

In the case of ceria, density functional theory (DFT) calculations have shown that the formation of an oxygen vacancy is energetically favored on the surfaces of ceria nanoparticles in comparison to extended ceria surfaces [7-9]. Indeed, experimental studies have revealed that nanoparticles of ceria have the capacity to support a high concentration of defects and reduced species, which are present in only minute quantities on planar surfaces [10-13]. Oxygen adsorption on ceria nanocrystallites of various morphologies was utilized to characterize the

difference in defect sites as a function of crystallographic orientation [14]. Defects on nanocrystalline ceria were found to be a controlling factor in the low-temperature ODH of ethylbenzene [15]. Also, the enhanced activity of ceria nanorods was reported for CO oxidation [16]. 
To shed light on the structure and reactivity of ceria-based catalysts, thin $\mathrm{CeO}_{2}(111)$ films grown on metal single crystals were used as planar model systems well-suited for vacuumbased, surface-sensitive techniques [17-19]. Ceria $\left(\mathrm{CeO}_{2}\right)$ crystallizes in the fluorite structure. When projected onto the (111) plane, the structure can be viewed as a stack of O-Ce-O trilayers with a surface lattice constant of $0.383 \mathrm{~nm}$. Fully oxidized $\mathrm{CeO}_{2}(111)$ is therefore terminated by an O-layer. DFT study of methanol adsorption on defect-free $\mathrm{CeO}_{2}(111)$ [20] suggested that dissociative adsorption of methanol by either $\mathrm{C}-\mathrm{H}$ or $\mathrm{O}-\mathrm{H}$ bond scission is more favorable than molecular adsorption, but only at low coverages. Another DFT calculation [21] showed that the dissociation of methanol may occur on the regular surface, but it is enhanced by the presence of a vacancy. An on-top methoxy species, where the methoxy oxygen binds to a cerium atom from the second layer, was identified as a dissociation product on the fully oxidized surface. On the partially reduced surface the dissociation product is a triply bridged methoxy species, where the methoxy oxygen partially fills the oxygen vacancy in the surface.

From experimental work it was concluded that methanol reacts with $\mathrm{CeO}_{2}$ even at low temperatures to produce water, which desorbs at $200 \mathrm{~K}$, and methoxy groups. The latter species have been characterized by infrared reflection-absorption spectroscopy (IRAS) as on top, doubly bridged, and triply bridged entities with respect to the Ce cations $[19,22-24]$. The surface methoxy species are quite stable and decompose at temperatures above $550 \mathrm{~K}$ to yield $\mathrm{H}_{2}, \mathrm{CO}, \mathrm{H}_{2} \mathrm{O}$, and formaldehyde (FA). On reduced ceria, more methanol can be adsorbed, and it undergoes more extensive decomposition to produce $\mathrm{CO}$ and $\mathrm{H}_{2}$ near $640 \mathrm{~K}$ in addition to $\mathrm{H}_{2} \mathrm{O}$ and FA. Ferrizz et al. [25], using a $\mathrm{CeO}_{2}(111)$ single crystal, argued that vacancies on the surface are necessary for methanol adsorption and dissociation, Mullins et al.[26] and Siokou and Nix [19] found that those processes also take place on the fully oxidized surface, although the dissociation of methanol on the surface is considerably enhanced in the presence of vacancies [21]. In addition, Ferrizz et al.[25] have examined $\sim 4 \mathrm{~nm}$-thick ceria films grown on the $\alpha$ $\mathrm{Al}_{2} \mathrm{O}_{3}(0001)$ and yttria-stabilized zirconia (100) substrates. The temperature programmed desorption (TPD) results were rationalized in terms of structural sensitivity of the methanol reaction on ceria. 
In this study, we investigate the methanol-to-FA reaction on ceria surfaces, in particular focusing on the reactivity of ceria nanoparticles for comparison with extended $\mathrm{CeO}_{2}(111)$ surfaces. The methanol adsorption was monitored by using TPD and IRAS. It is revealed that the ceria nanoparticles exhibit low-temperature activity that does not exist on the ordered planar surface.

\section{Experimental}

The experiments were performed in an ultrahigh vacuum (UHV) chamber with a base pressure of $\sim 5 \times 10^{-10}$ mbar. The chamber is equipped with an IR spectrometer (Bruker IFS 66/vs), a differentially pumped quadrupole mass spectrometer (Hiden HAL 201), and a lowenergy electron diffraction/Auger electron spectroscopy apparatus (LEED/AES, from Specs). The $\mathrm{Ru}(0001)$ crystal (10 $\mathrm{mm}$ in diameter, $2 \mathrm{~mm}$ in thickness) was spot-welded to two parallel Ta wires that were in turn welded to two Ta rods used for resistive heating and also for cooling by filling a manipulator tube with liquid nitrogen. The temperature was measured by a chromelalumel thermocouple spot-welded to the backside of the crystal.

Thin, well-ordered $\mathrm{SiO}_{2}$ films were grown on a $\mathrm{Ru}(0001)$ single-crystal substrate as described elsewhere $[27,28]$. Briefly, the clean $\mathrm{Ru}(0001)$ was precovered with oxygen to form a 30 (2×2)-layer. Then, silicon (99.95\%, from Goodfellow) was vapor-deposited in $2 \times 10^{-7} \mathrm{mbar}_{2}$ at a substrate temperature of $\sim 100 \mathrm{~K}$, and subsequently annealed at $\sim 1240 \mathrm{~K}$ in $2 \times 10^{-6} \mathrm{mbar}_{2}$ for $15 \mathrm{~min}$. Note that these so-called bilayer films are weakly bound to the underlying Ru support, that is in contrast to the previously used monolayer silica films grown on Mo(112) [13].

Ceria particles were deposited on the $\mathrm{SiO}_{2}$ surface by using an ice-assisted deposition method [13]. First, about $80 \mathrm{~L} \mathrm{(} 1 \mathrm{~L}=10^{-6}$ Torr s) of $\mathrm{D}_{2} \mathrm{O}$ was dosed onto the $\mathrm{SiO}_{2}$ surface at $100 \mathrm{~K}$ to create a thick layer of ice on the surface, which was confirmed with IRAS. The ceria was evaporated upon this ice layer at $100 \mathrm{~K}$ and $10^{-6} \mathrm{mbar} \mathrm{O}_{2}$ for various times from a tungsten crucible contained in an e-beam-assisted evaporator. After Ce-deposition, the substrate was slowly heated to $300 \mathrm{~K}$ in $10^{-6} \mathrm{mbar}_{2}$ to desorb the ice layer, which occurs at $\sim 160-180 \mathrm{~K}$ [29]. Then, the sample was annealed in $10^{-6} \mathrm{mbar}_{2}$ at $715 \mathrm{~K}$ for $5 \mathrm{~min}$ to create a stable morphology for TPD studies. 
Methanol (Merck, 99.8\% purity) was purified by utilizing repeated freeze-pump-thaw cycles and then exposed to the sample by using a pinhole gas doser located approximately $2 \mathrm{~cm}$ from the sample surface. The magnitude of the dose was approximately $25 \mathrm{~L}$ and the sample was held at room temperature during dosing to inhibit methanol physisorption that would obscure reactivity at low temperatures in the TPD spectra. The sample was cooled to $100 \mathrm{~K}$ prior to TPD measurement and then heated to $700 \mathrm{~K}$ at a rate of $3 \mathrm{Ks}^{-1}$. The TPD signal for $\mathrm{m} / \mathrm{z}=$ 29 is the major component of the FA cracking pattern and was used to determine the degree of FA formation. To correct this signal for the desorption of methanol (which also has a component at $\mathrm{m} / \mathrm{z}=29$ ) the following equation was used $I^{F A}=\mathrm{I}^{\mathrm{m} / z=29}-A \times \mathrm{I}^{\mathrm{m} / z}=31$, for which the correction factor $A$ was empirically determined for our measurement system using the desorption of a multilayer of physisorbed methanol.

For comparison to the $\mathrm{CeO}_{x}$ particle samples, 5-nm thick crystalline $\mathrm{CeO}_{2}(111) / \mathrm{Ru}(0001)$ films were grown as described elsewhere $[13,18,26]$. It has been previously shown that $\mathrm{CeO}_{2}(111)$ films expose broad terraces and a low concentration of defects.

Additional characterization of similarly prepared ceria/ $/ \mathrm{SiO}_{2}$ samples was performed in another UHV chamber equipped with a scanning tunneling microscope (STM), X-ray photoelectron spectroscopy (XPS), and IRAS, the latter being used to link the structural data obtained in two experimental setups.

\section{Results and Discussion}

We first address the structural characteristics of the ceria deposits. For deposition onto a clean $\mathrm{SiO}_{2}$ film in oxygen ambient the ceria was present primarily in the oxidation state 4+, although certain amounts of $\mathrm{Ce}^{3+}$ were also found in the Ce3d XP-spectra, which were similar to previously reported spectra of ceria nanoparticles deposited onto monolayer silica films grown on $\mathrm{Mo}(112)$ [13]. Deposition onto an ice-precovered silica/Ru(0001) surface and oxidation at elevated temperatures $(\sim 720 \mathrm{~K})$ resulted in fully oxidized ceria as judged by XPS. At increasing Ce coverage the silica-related signals (i.e., Si2p at $\sim 102.5 \mathrm{eV}$, and $01 \mathrm{~s}$ at $\sim 532 \mathrm{eV}$ ) were gradually attenuated, whereas the $01 \mathrm{~s}$ signal at $\sim 529.5 \mathrm{eV}$ gained intensity and is therefore 
assigned to oxygen in ceria. LEED inspection revealed only attenuation of the substrate diffraction spots. IRA-spectra of the resulting ceria/ $/ \mathrm{SiO}_{2}$ surfaces revealed attenuation and a red-shift of the principal phonon of the silica film (at $\sim 1300 \mathrm{~cm}^{-1}$ ) without any additional spectral features. It is therefore concluded that the interaction of ceria with silica films under these conditions does not destroy the ordering of the $\mathrm{SiO}_{2}$ film as observed, for instance, for Fesubstituted silicate films [30]. Indeed, STM images revealed the formation of ceria nanoparticles $\sim 2 \mathrm{~nm}$ in size, as displayed in Fig. 1 for the lowest Ce coverage studied. The STMcalibrated spectral changes in IRAS upon ceria deposition were used to have an estimate of the ceria coverage in the other UHV chamber that was used for methanol adsorption studies.

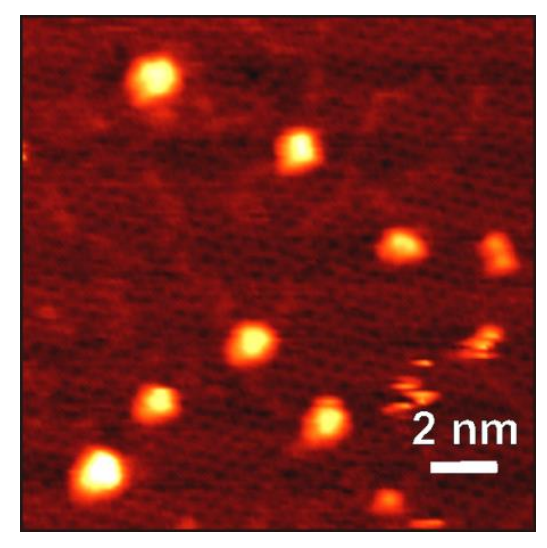

Figure 1. STM image of ceria particles formed on a well-ordered silica film at low coverages. Tunneling conditions: the sample bias $8 \mathrm{~V}$, the current $0.1 \mathrm{nA}$.

The adsorption results below are primarily presented for three ceria coverages, which caused red-shifts of the $1300 \mathrm{~cm}^{-1}$ silica phonon by approximately 6,13 , and $24 \mathrm{~cm}^{-1}$. The coverage was varied by the Ce deposition time and correspond roughly to $0.25,0.5$, and 1 monolayer $(\mathrm{ML})$ with respect to the $\mathrm{Ru}(0001)$ surface atoms $\left(1 \mathrm{ML}=6.4 \times 10^{14} \mathrm{at} / \mathrm{cm}^{2}\right)$. These are henceforth referred to as low, medium, and high coverage, respectively. 


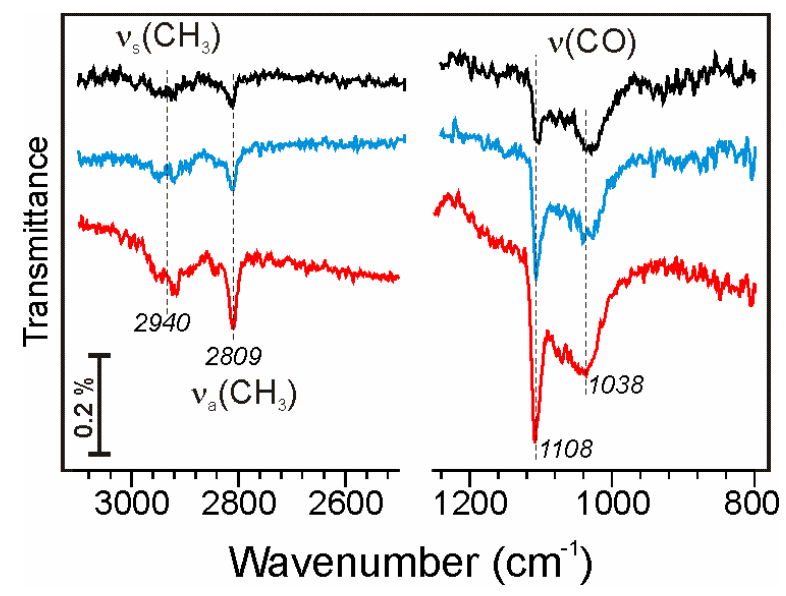

Figure 2. IRA spectra of methanol adsorbed on $\mathrm{CeO}_{x} / \mathrm{SiO}_{2}$ films at increasing ceria coverage (from top to bottom). The methanol dosing was performed at $300 \mathrm{~K}$, and the samples were subsequently cooled to $100 \mathrm{~K}$ for measurements.

Figure 2 shows the $v(\mathrm{CO})$ and $v\left(\mathrm{CH}_{3}\right)$ regions in the IRA spectra of $\mathrm{CeO}_{x} / \mathrm{SiO}_{2}$ films after methanol adsorption at $300 \mathrm{~K}$ and cooling down to $100 \mathrm{~K}$ as a function of ceria coverage. All observed bands are characteristic of methoxy formation on the ceria surface $[19,22-24]$. The bands in the $\mathrm{CO}$ stretch region are commonly identified in terms of methoxy groups adsorbed in atop (1105-1115 cm $\left.\mathrm{cm}^{-1}\right)$, bridging $\left(1060-1080 \mathrm{~cm}^{-1}\right)$, and tridentate $\left(1015-1045 \mathrm{~cm}^{-1}\right)$ geometries with respect to the $\mathrm{Ce}^{4+}$ ions. Accordingly, our spectra clearly show peaks at $1108 \mathrm{~cm}^{-1}$ and 1038 $\mathrm{cm}^{-1}$ that can be assigned to mono-, and tri-dentate species, albeit some intensity between these two bands could be indicative of bi-dentate species as well. All bands gain intensity with increasing ceria coverage, but the $1108 \mathrm{~cm}^{-1}$ component shows a higher relative intensity for the higher coverages. The $\mathrm{C}-\mathrm{H}$ stretch region is less sensitive to the adsorption geometry, therefore, both the symmetric $v_{s}\left(C_{3}\right)$ band at $\sim 2810 \mathrm{~cm}^{-1}$ and asymmetric $v_{a}\left(\mathrm{CH}_{3}\right)$ band at $2930 \mathrm{~cm}^{-1}$ simply scale with the ceria loading. A more quantitative analysis is hardly possible here without precise knowledge about the ceria morphology which, in turn, determines the orientation of adsorbed species with respect to the surface plane and hence signal intensity due to the well-known metal selection rules in IRAS.

The same samples were then studied by using TPD. Only masses related to the cracking patterns of methanol and FA in the mass spectrometer were observed in the TPD spectra as depicted in Fig. 3. The desorption signals at $\mathrm{T}<300 \mathrm{~K}$ are associated with the adsorption of 
residual methanol from the UHV background upon cooling to $100 \mathrm{~K}$ for IRAS measurements. Note that the desorption profile of methanol commonly shows a long descending tail due to the low pumping speed of alcohols (and water). The methanol curves in these spectra are essentially featureless, except for the high-coverage sample, which shows some desorption at approximately $575 \mathrm{~K}$ that is additionally accompanied by the desorption of water. In contrast, the FA signals showed a considerable dependence upon ceria coverage (note that pure silica films did not show any reactivity towards methanol).

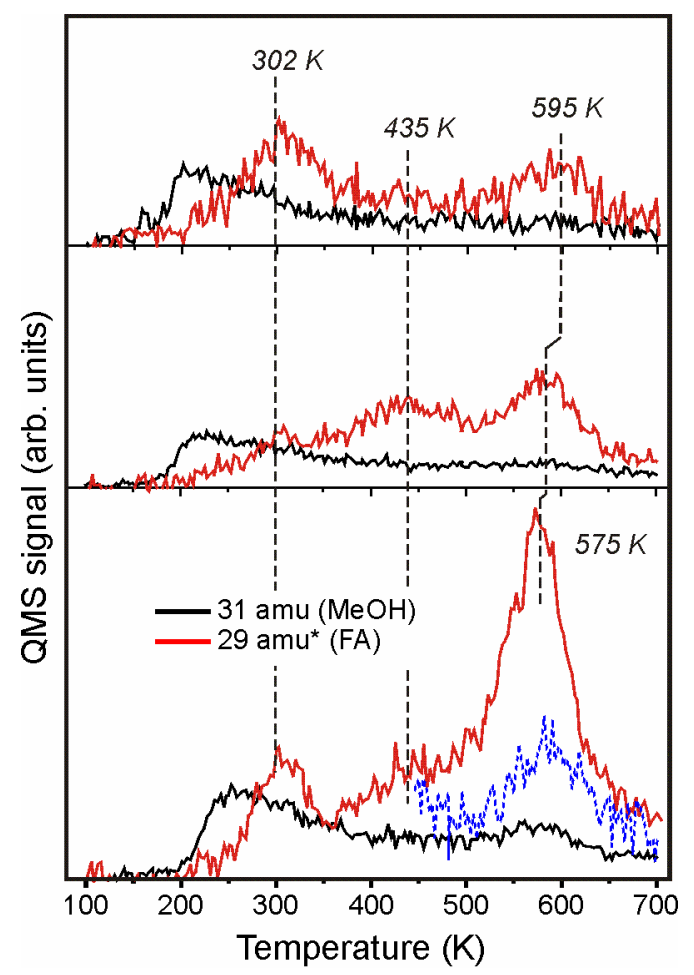

Figure 3. TPD spectra of methanol adsorbed at $300 \mathrm{~K}$ on $\mathrm{CeO}_{x} / \mathrm{SiO}_{2}$ films at increasing ceria coverages (from top to bottom). The pairs of curves show the $31 \mathrm{amu}$ (methanol) desorption signal and fragmentation-corrected 29 amu signal of formaldehyde (see Experimental). The blue dashed line in the bottom panel additionally shows the $18 \mathrm{amu}\left(\mathrm{H}_{2} \mathrm{O}\right)$ signal, which could be detected only for samples at high ceria coverage. The signals at $\mathrm{T}<300 \mathrm{~K}$ are associated with adsorption of residual methanol from the UHV background upon cooling to $100 \mathrm{~K}$.

For the low-coverage sample, two desorption peaks are observed (i.e., at 305 and 595 K). For the high-coverage sample, the high-temperature signal dominates the spectrum peaked at $575 \mathrm{~K}$, which is close to that observed for $\mathrm{CeO}_{2}(111)$ surfaces $[19,25,26,31]$, and indicates 
that the ceria is becoming more "film-like" at high coverages. The supported nanoparticles also show FA production at around $435 \mathrm{~K}$, which is more clearly seen for the medium-coverage sample. The relative intensity of the low-temperature activity with respect to the conventional high-temperature peak is greater for the lower ceria coverages, which indicates that this may be a size effect.
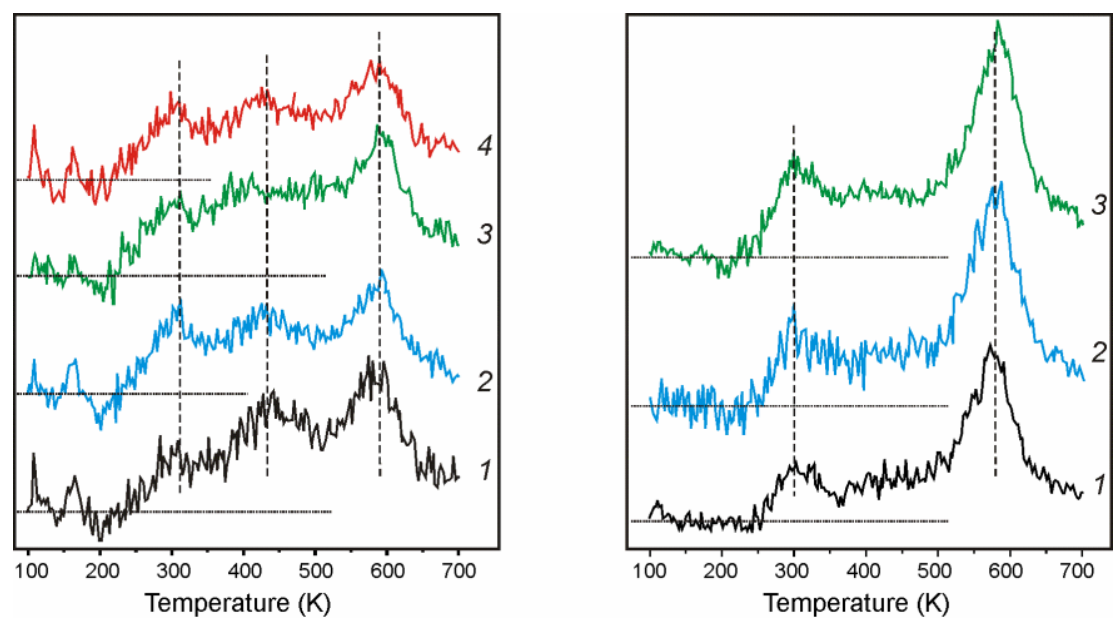

Figure 4. Formaldehyde signals in TPD spectra of methanol adsorbed at $300 \mathrm{~K}$ on two samples with medium (on the left) and high (on the right) ceria coverages. The "as prepared" sample (spectrum 1) was reoxidized after the first TPD run and spectrum (2) was recorded following methanol adsorption at $300 \mathrm{~K}$. Spectrum (3) is obtained without reoxidation step. Finally, the sample was reoxidized again to record spectrum (4). The spectra are offset for clarity.

Repeated TPD runs for the medium- and high-coverage samples are shown in Fig. 4. Comparison of the subsequent TPD spectra reveals basically the same desorption profiles, irrespective of whether or not the sample was reoxidized (under the same conditions as used for the ceria preparation, i.e., $10^{-6} \mathrm{mbar}_{2}, 715 \mathrm{~K}, 5 \mathrm{~min}$ ) after the previous experiment. The results for the low-coverage sample showed similar behavior, at least for the high-temperature FA production. The typical mechanism of the methanol reaction on a ceria surface involves the creation of an oxygen vacancy which should be regenerated by reoxidation or other rearrangement to create a full catalytic cycle. It has been observed previously on $\mathrm{CeO}_{2}(111)$ single-crystal surfaces that the reaction of methanol on ceria tends to favor the complete hydrogenation to $\mathrm{CO}$ after the completion of multiple TPD runs, which create a high 
concentration of $\mathrm{Ce}^{3+}$ sites at the surface [25]. In the case of nano-ceria, it appears that this oxidation step is not necessary to regenerate the activity, at least over the duration of one TPD run. This finding may indicate that ceria nanoparticles possess oxygen capacity sufficient for the reaction to occur during several TPD runs.

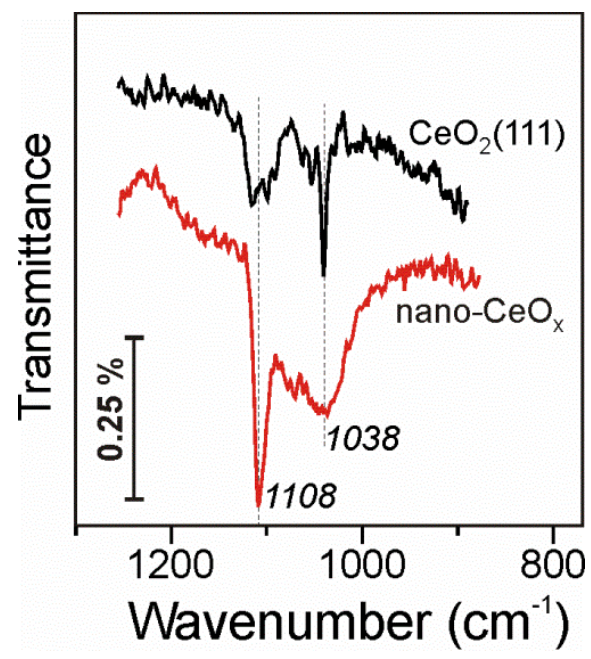

Figure 5. CO stretch region in IRA spectra of methoxy species formed upon methanol adsorption at 300 $\mathrm{K}$ on the well-ordered $\mathrm{CeO}_{2}(111)$ film (top) and the $\mathrm{SiO}_{2}$-supported $\mathrm{CeO}_{x}$ particles at high coverage (bottom), both showing FA production at $\sim 570 \mathrm{~K}$ in the TPD spectra of methanol.

To compare to the ceria-particle samples, an ordered $\mathrm{CeO}_{2}(111)$ film was grown on the same $\mathrm{Ru}(0001)$ substrate that was used for growth of the silica film, and the reactivity was probed with methanol in the same way as on the $\mathrm{CeO}_{\mathrm{x}} / \mathrm{SiO}_{2}$ samples. Figure 5 shows IRA spectra after methanol adsorption, comparing an ordered $\mathrm{CeO}_{2}(111)$ film and nano- $-\mathrm{CeO}_{x} / \mathrm{SiO}_{2}$. The same two peaks were observed at $1108 \mathrm{~cm}^{-1}$ and $1038 \mathrm{~cm}^{-1}$, which varied in broadness and intensity. In contrast to the well-ordered ceria film, the supported ceria particles showed the $1108 \mathrm{~cm}^{-1}$ signal more intense than the signal at lower frequency.

TPD spectra on the ordered $\mathrm{CeO}_{2}(111)$ film showed FA desorption at $565 \mathrm{~K}$ (not shown here), which is in excellent agreement with that observed in our previous study [31], but considerably lower than that observed above on the ceria nanoparticles (575 - $595 \mathrm{~K}$, see Fig. 3). This fits well the general trend revealed in Fig. 3 that the high-temperature FA peak shifts to a lower temperature at increasing particle size, ultimately up to a single crystal. This finding 
indicates that respective methoxy species react towards FA more easily on extended (111) surfaces. On the other hand, the low-temperature $(<450 \mathrm{~K})$ activity is characteristic only for the nanoparticle systems, which implies the presence of another methoxy species giving rise to FA at a lower temperature. Figure 5 suggests that there is no straightforward relationship between the adsorption geometry of methoxy species and their reactivity towards FA. We may only speculate that a relatively higher intensity of the $1108 \mathrm{~cm}^{-1}$ band on particles, commonly assigned to methoxy in atop geometry and which, on the basis of DFT calculations [21], is less stable compared to adsorption on oxygen vacancy, would be consistent with the methanol adsorption on low-coordinated Ce sites present on the particle edges. In addition, the energy of formation of an oxygen vacancy on the surfaces of nanoparticles has been shown to be significantly lower than that of planar surfaces, which leads to much higher oxygen-vacancy concentrations in the nanoparticles, and this property is strongly dependent upon size for small particles [7-9]. Moreover, the most energetically favored oxygen vacancies are created at edge sites [8]. The additional low-temperature formaldehyde production observed on nanoparticle samples in our study could likely be due to new reaction pathways opened up by the weakly bound oxygen species involved in the methoxy transformation into FA.

Another possible explanation comes from the fact that ceria nanoparticles can expose other surfaces in addition to the (111) facet. On the basis of DFT calculations and the GibbsWulf theorem of equilibrium crystal shape Fronzi et al. [32] predicted the morphology of unsupported $\mathrm{CeO}_{2}$ particles to be dominated by the (111) facets dominate the morphology under both oxygen-rich and oxygen-lean conditions. In the latter case, the particles may expose (100) facets as well. Also, Neyman and coworkers [7-9] considered octahedral and cubooctahedral particle shapes to be the most stable. Certainly, the morphology of the silicasupported ceria nanoparticles studied here is ill-defined and, in fact, difficult to determine precisely. However, assuming a weak interaction of ceria with a silica-film support as judged by IRAS, we propose that the particle morphology is somewhat similar to that of unsupported ceria, and is thus dominated by (111) facets. If so, the observed low-temperature reactivity must be attributed to the under-coordinated surface atoms rather than the specific reactivity of the (100) facets with respect to methanol. 
Finally, it should be mentioned that low-temperature FA production was previously observed on small vanadyl $(\mathrm{V}=\mathrm{O})$ clusters supported on $\mathrm{CeO}_{2}(111)$ film, which was proposed to be related to both the high dispersion of the vanadia particles as well as the degree of reduction of the ceria support in the vicinity of the vanadyl species [31]. It seems plausible that the ceria nanoparticles deposited in this study have stabilized similar species without the presence of vanadia. It is therefore anticipated that the nano-ceria system would be interesting to explore under more realistic conditions in the $\mathrm{ODH}$ reactions.

\section{Conclusions}

The reactivity of methanol on various ceria surfaces was probed by using IRAS and the formaldehyde production was monitored by using TPD. TPD results showed low-temperature reactivity $(400-450 \mathrm{~K})$ for silica-supported $\mathrm{CeO}_{x}$ particles, which is not present on well-ordered $\mathrm{CeO}_{2}(111)$ films. The TPD and IRAS observations together show that low-coordinated sites, presumably at particle edges, play an important role in methanol reactivity on ceria nanoparticles.

\section{Acknowledgements}

We acknowledge the support from the COST Action CM1104 "Reducible oxide chemistry, structure and functions". 


\section{References}

1. Bernal, S., J. Kaspar, and A. Trovarelli, Recent progress in catalysis by ceria and related compounds - Preface. Catalysis Today, 1999. 50(2): p. 173-173.

2. Trovarelli, A., Catalytic properties of ceria and CeO2-containing materials. Catalysis ReviewsScience and Engineering, 1996. 38(4): p. 439-520.

3. Jen, H.W., et al., Characterization of model automotive exhaust catalysts: Pd on ceria and ceriazirconia supports. Catalysis Today, 1999. 50(2): p. 309-328.

4. Wachs, I.E., Recent conceptual advances in the catalysis science of mixed metal oxide catalytic materials. Catalysis Today, 2005. 100(1-2): p. 79-94.

5. Sauer, J. and J. Dobler, Structure and reactivity of V2O5: bulk solid, nanosized clusters, species supported on silica and alumina, cluster cations and anions. Dalton Transactions, 2004(19): p. 3116-3121.

6. Ganduglia-Pirovano, M.V., et al., Role of Ceria in Oxidative Dehydrogenation on Supported Vanadia Catalysts. Journal of the American Chemical Society, 2010. 132(7): p. 2345-2349.

7. Migani, A., et al., Greatly facilitated oxygen vacancy formation in ceria nanocrystallites. Chemical Communications, 2010. 46(32): p. 5936-5938.

8. Migani, A., et al., Dramatic reduction of the oxygen vacancy formation energy in ceria particles: $a$ possible key to their remarkable reactivity at the nanoscale. Journal of Materials Chemistry, 2010. 20(46): p. 10535-10546.

9. Loschen, C., et al., Density functional studies of model cerium oxide nanoparticles. Physical Chemistry Chemical Physics, 2008. 10(37): p. 5730-5738.

10. Wang, A.Q., et al., X-ray photoelectron spectroscopy study of electrodeposited nanostructured $\mathrm{CeO}[s u b$ 2] films. Journal of Vacuum Science \& Technology B: Microelectronics and Nanometer Structures, 2003. 21(3): p. 1169-1175.

11. Tsunekawa, S., T. Fukuda, and A. Kasuya, $X$-ray photoelectron spectroscopy of monodisperse CeO2-x nanoparticles. Surface Science, 2000. 457(3): p. L437-L440.

12. Qiu, L., et al., Comparative XPS study of surface reduction for nanocrystalline and microcrystalline ceria powder. Applied Surface Science, 2006. 252(14): p. 4931-4935.

13. Baron, M., et al., Interaction of Gold with Cerium Oxide Supports: CeO2(111) Thin Films vs CeOx Nanoparticles. The Journal of Physical Chemistry C, 2009. 113(15): p. 6042-6049.

14. Wu, Z.L., et al., Probing Defect Sites on CeO2 Nanocrystals with Well-Defined Surface Planes by Raman Spectroscopy and O-2 Adsorption. Langmuir. 26(21): p. 16595-16606.

15. Murugan, B. and A.V. Ramaswamy, Defect-site promoted surface reorganization in nanocrystalline ceria for the low-temperature activation of ethylbenzene. Journal of the American Chemical Society, 2007. 129(11): p. 3062-+.

16. Zhou, K., et al., Enhanced catalytic activity of ceria nanorods from well-defined reactive crystal planes. Journal of Catalysis, 2005. 229(1): p. 206-212.

17. Kuhlenbeck, H., S. Shaikhutdinov, and H.-J. Freund, Well-Ordered Transition Metal Oxide Layers in Model Catalysis - A Series of Case Studies. Chemical Reviews, 2013. 113(6): p. 3986-4034.

18. Mullins, D.R., P.V. Radulovic, and S.H. Overbury, Ordered cerium oxide thin films grown on $R u(0001)$ and $\mathrm{Ni}(111)$. Surface Science, 1999. 429(1-3): p. 186-198.

19. Siokou, A. and R.M. Nix, Interaction of Methanol with Well-Defined Ceria Surfaces: Reflection/Absorption Infrared Spectroscopy, X-ray Photoelectron Spectroscopy, and Temperature-Programmed Desorption Study. The Journal of Physical Chemistry B, 1999. 103(33): p. 6984-6997. 
20. Mei, D., et al., Methanol Adsorption on the Clean CeO2(111) Surface: A Density Functional Theory Study. The Journal of Physical Chemistry C, 2007. 111(28): p. 10514-10522.

21. Beste, A., et al., Adsorption and dissociation of methanol on the fully oxidized and partially reduced (1\&\#xa0;1\&\#xa0;1) cerium oxide surface: Dependence on the configuration of the cerium 4 f electrons. Surface Science, 2008. 602(1): p. 162-175.

22. Badri, A., C. Binet, and J.-C. Lavalley, Use of methanol as an IR molecular probe to study the surface of polycrystalline ceria. Journal of the Chemical Society, Faraday Transactions, 1997. 93(6): p. 1159-1168.

23. Li, C., et al., Spectroscopic identification of adsorbed species derived from adsorption and decomposition of formic acid, methanol, and formaldehyde on cerium oxide. Journal of Catalysis, 1990. 125(2): p. 445-455.

24. Binet, C., M. Daturi, and J.-C. Lavalley, IR study of polycrystalline ceria properties in oxidised and reduced states. Catalysis Today, 1999. 50(2): p. 207-225.

25. Ferrizz, R.M., et al., Structure Sensitivity of the Reaction of Methanol on Ceria. Langmuir, 2001. 17(8): p. 2464-2470.

26. Mullins, D.R., M.D. Robbins, and J. Zhou, Adsorption and reaction of methanol on thin-film cerium oxide. Surface Science, 2006. 600(7): p. 1547-1558.

27. Löffler, D., et al., Growth and Structure of Crystalline Silica Sheet on Ru(0001). Physical Review Letters, 2010. 105(14): p. 146104.

28. Yang, B., et al., Thin silica films on Ru(0001): monolayer, bilayer and three-dimensional networks of [SiO4] tetrahedra. Physical Chemistry Chemical Physics, 2012.

29. Yang, B., et al., Hydroxylation of Metal-Supported Sheet-Like Silica Films. The Journal of Physical Chemistry C, 2013. 117(16): p. 8336-8344.

30. Wlodarczyk, R., et al., The atomic structure of an ultrathin Fe-silicate film grown on a metal: $A$ monolayer of clay? . under review in JACS, 2013.

31. Abbott, H.L., et al., Relating methanol oxidation to the structure of ceria-supported vanadia monolayer catalysts. Journal of Catalysis, 2010. 272(1): p. 82-91.

32. Fronzi, M., et al., Stability and morphology of cerium oxide surfaces in an oxidizing environment: A first-principles investigation. The Journal of Chemical Physics, 2009. 131(10): p. 104701-16. 